\title{
The knockout of predicted MYB60 gene in Eruca sativa promotes anthocyanin accumulation
}

\author{
Khusnutdinov E., Panfilova M., Sukhareva S., Mikhaylova E.* \\ Institute of biochemistry and genetics UFRC RAS, Ufa, Russia \\ *email:mikhele@list.ru
}

Eruca sativa (E. vesicaria, rocket) is a valuable plant in the family Brassicaceae, used as a leaf vegetable. Its genome is represented only by one assembly (GCA_902460325.1) which is not annotated. The functions of E. sativa genes are not determined yet.

Transcription factor MYB60 is a well known negative regulator of anthocyanin biosynthesis in Brassicaceae, including Arabidopsis thaliana, Brassica rapa, Brassica napus, Brassica oleracea, etc. Variation in the expression level of MYB60 gene affects anthocyanin biosynthesis. Therefore mutants are visible due to increased or decreased pigmentation, which can serve as a great model system to develop transformation methods and genetic constructs. Leaf vegetables accumulating anthocyanins can become popular as a component of a healthy diet. Unfortunately, homologs of this gene and encoded protein in E. sativa have never been identified. By our experience, this species can be successfully transformed via floral dip method, just like $A$. thaliana. It makes E. sativa a noteworthy experimental object for genome editing.

gRNA spacer was selected for the most conserved region of the gene and cloned into the pDIRECT_22A vector (Addgene plasmid \# 91133). Four plants were treated with Agrobacterium tumefaciens carrying the plasmid, and 150 resultant seeds were sown. Among seedlings five putative mutants with pigmented leaves and flowers and/or decreased stem length were chosen for further analysis. Expression level of predicted MYB60 gene in two dwarf and pigmented mutants was 5 times lower than in wild type plant. Melting curve analysis revealed that both of them were probably homozygous mutants. Three putative mutants had normal stem length and 3 -fold reduction in gene expression. According to melting curve analysis, they were either heterozygous of chimeric. These results suggest that the putative homolog of MYB60 gene of E. sativa has the same functions and can be used as a target to increase anthocyanin accumulation. Acknowledgments: The reported study was funded by Russian Science Foundation according to the research project No. 20-74-10053. 